\title{
Slices of the unitary spread
}

\section{G. Lunardon · L. Parlato $\cdot$ V. Pepe $\cdot$ R. Trombetti}

Received: 17 April 2009 / Accepted: 14 April 2010 / Published online: 4 May 2010

(C) Springer Science+Business Media, LLC 2010

\begin{abstract}
We prove that slices of the unitary spread of $\mathcal{Q}^{+}(7, q), q \equiv 2(\bmod 3)$, can be partitioned into five disjoint classes. Slices belonging to different classes are non-equivalent under the action of the subgroup of $\mathrm{P \Gamma} \mathrm{O}^{+}(8, q)$ fixing the unitary spread. When $q$ is even, there is a connection between spreads of $\mathcal{Q}^{+}(7, q)$ and symplectic 2-spreads of PG $(5, q)$ (see Dillon, Ph.D. thesis, 1974 and Dye, Ann. Mat. Pura Appl. (4) 114, 173-194, 1977). As a consequence of the above result we determine all the possible non-equivalent symplectic 2-spreads arising from the unitary spread of $\mathcal{Q}^{+}(7, q), q=2^{2 h+1}$. Some of these already appeared in Kantor, SIAM J. Algebr. Discrete Methods 3(2), 151-165, 1982. When $q=3^{h}$, we classify, up to the action of the stabilizer in $P \Gamma O(7, q)$ of the unitary spread of $Q(6, q)$, those among its slices producing spreads of the elliptic quadric $\mathcal{Q}^{-}(5, q)$.
\end{abstract}

Keywords Ovoid · Unitary spread · Slice

G. Lunardon · L. Parlato $\cdot$ R. Trombetti $(\bowtie)$

Dipartimento di Matematica e Applicazioni "R. Caccioppoli”, Università degli Studi di Napoli

"Federico II", 80126 Napoli, Italy

e-mail: rtrombet@unina.it

G. Lunardon

e-mail: lunardon@unina.it

L. Parlato

e-mail: laura.parlato@dma.unina.it

V. Pepe

Department of Pure Mathematics and Computer Algebra, University of Gent, Krijgslaan 281, 9000

Gent, Belgium

e-mail: valepepe@cage.ugent.be 


\section{Introduction}

Let $\mathcal{Q}=\mathcal{Q}^{+}(7, q)$ be the hyperbolic quadric of $\mathrm{PG}(7, q)$. A spread $\mathcal{S}$ of $\mathcal{Q}$ is a partition of the pointset of $\mathcal{Q}$ into 3-dimensional totally singular subspaces. Denote by $\mathbb{M}_{1}$ and $\mathbb{M}_{2}$ the two families of maximal singular subspaces of $\mathcal{Q}$ (for definition and details see [14]). Then the spread $\mathcal{S}$ consists of $q^{3}+1$ subspaces of $\mathcal{Q}$ belonging either to $\mathbb{M}_{1}$ or to $\mathbb{M}_{2}$. Two spreads $\mathcal{S}$ and $\mathcal{S}^{\prime}$ of $\mathcal{Q}$ are said to be isomorphic if there is a collineation of $P \Gamma O^{+}(8, q)$ mapping any element of $\mathcal{S}$ into an element of $\mathcal{S}^{\prime}$.

An ovoid of $\mathcal{Q}$ is a set of points of $\mathcal{Q}$ which has exactly one point in common with every 3-dimensional totally singular subspace of $\mathcal{Q}$; an ovoid of $\mathcal{Q}$ consists of $q^{3}+1$ points. Two ovoids $\mathcal{O}$ and $\mathcal{O}^{\prime}$ of $\mathcal{Q}$ are said to be isomorphic if there is a collineation of $P \Gamma O^{+}(8, q)$ mapping $\mathcal{O}$ into $\mathcal{O}^{\prime}$.

Ovoids and spreads of $\mathcal{Q}$ are related by triality. Precisely, let $\mathcal{P}$ be the point set of $\mathcal{Q}$ and let $\mathcal{L}$ be the set of all lines contained in $\mathcal{Q}$. A triality map $\tau$ of $\mathcal{Q}$ is a map of order 3 such that $\tau: \mathcal{L} \rightarrow \mathcal{L}$ and $\tau: \mathcal{P} \rightarrow \mathbb{M}_{1} \rightarrow \mathbb{M}_{2} \rightarrow \mathcal{P}$, which preserves the incidence between members of $\mathcal{L}$ and members of $\mathcal{P} \cup \mathbb{M}_{1} \cup \mathbb{M}_{2}$ (see [16, 17]). Hence, if we set $\mathcal{S} \subset \mathbb{M}_{1}$, then $O=\mathcal{S}^{\tau^{2}}$ is an ovoid of $\mathcal{Q}$, and conversely.

Let, now, $\Pi$ be any non-singular hyperplane of $\operatorname{PG}(7, q)$, then the set $\mathcal{S}^{\prime}=\{\Pi \cap$ $S: S \in \mathcal{S}\}$ defines a spread of the parabolic quadric $\mathcal{Q}^{\prime}=\mathcal{Q}(6, q)=\Pi \cap \mathcal{Q}$, i.e. a set of $q^{3}+1$ singular planes partitioning the points of $\mathcal{Q}^{\prime}$. We refer to these spreads as the slices of the spread $\mathcal{S}$. Conversely, start from a spread $\mathcal{S}^{\prime}$ of a parabolic quadric $\mathcal{Q}^{\prime}=\mathcal{Q}(6, q)$ of $\operatorname{PG}(6, q)$; and embed $\mathcal{Q}^{\prime}$ as a non-singular hyperplane section of the hyperbolic quadric $\mathcal{Q}=\mathcal{Q}^{+}(7, q)$. For any spread element consider the totally singular 3-dimensional space of $\mathcal{Q}$, of fixed type, passing through it. This set of 3dimensional subspaces is a spread of $\mathcal{Q}$ and $\mathcal{S}^{\prime}$ is one of its slices.

A 2-spread of the projective space $P=\operatorname{PG}(5, q)$ is a family $\mathcal{S}$ of mutually disjoint planes partitioning the pointset of $P$. The spread $\mathcal{S}$ is said to be symplectic with respect to a symplectic polarity of $P$ if all elements of $\mathcal{S}$ are totally isotropic with respect to this symplectic polarity. We denote by $W(5, q)$ the polar space associated with a symplectic polarity of $\operatorname{PG}(5, q)$. Since when $q$ is even the parabolic quadric $\mathcal{Q}^{\prime}$ is isomorphic to a symplectic space $W(5, q)$, there is a connection between spreads of the hyperbolic quadric $\mathcal{Q}$ and 2-spreads of $W(5, q)$ and vice-versa. This was originally pointed out by Dillon [3] and Dye [4]. Moreover, if two 2-spreads of $W(5, q)$ are isomorphic (i.e. equivalent under the action of $P \Gamma \operatorname{Sp}(6, q)$ ), then the associated spreads of $\mathcal{Q}$ also are. The converse is not generally true (see [9]). This fact leads to the following definition in [9]: two 2-spreads of $W(5, q), q$ even, are said to be cousins if the associated spreads in the hyperbolic quadric $Q^{+}(7, q)$, obtained as described above, are equivalent. In the light of this fact one can construct all cousins of a given 2-spread of $W(5, q)$ by slicing a spread of a hyperbolic quadric; i.e. in the following way: construct the spread of $\mathcal{Q}^{+}(7, q)$ associated with the starting 2-spread in $W(5, q)$, then consider its various slices. As suggested in [9], we are only interested in those cousins that are not isomorphic under the action of the stabilizer of $\mathcal{S}$ in the orthogonal group $P \Gamma \mathrm{O}^{+}(8, q)$. Similarly, one can define the slice of an ovoid of $\mathcal{Q}$ or of an ovoid of $\mathcal{Q}^{\prime}$.

\footnotetext{
${ }^{1}$ An ovoid of $\mathcal{Q}^{\prime}=\mathcal{Q}(6, q)$ is a set of points of $\mathcal{Q}^{\prime}$ which has exactly one point in common with every totally singular plane of $\mathcal{Q}^{\prime}$. Also in this case, an ovoid has $q^{3}+1$ points.
} 
In [9] and [8], Kantor defines the so called unitary ovoid and unitary spread of $\mathcal{Q}$ and $\mathcal{Q}^{\prime}$, when $q \equiv 2(\bmod 3)$ and $q \equiv 0(\bmod 3)$, respectively; although, the unitary ovoid for $q \equiv 2(\bmod 3)$ already appeared in [16]. The stabilizers of both these geometric objects contain, up to isomorphism, the projective unitary group $\operatorname{PGU}(3, q)$. Also, when $q \equiv 0(\bmod 3)$, the parabolic quadric $\mathcal{Q}^{\prime}$ can be embedded in a hyperbolic quadric and the unitary ovoid of $\mathcal{Q}^{\prime}$ is an ovoid of this hyperbolic quadric, as well. Nevertheless, the unitary spread of $\mathcal{Q}^{\prime}$ defines a spread of the relevant hyperbolic quadric also permuted by $\operatorname{PGU}(3, q)$ [9, Theorem 6.14]. For $q=3^{2 h+1}$ this latter spread already appeared in [15].

Unitary spread and unitary ovoid, seen as geometric objects of a hyperbolic quadric, are related to each other by a triality map of the hyperbolic quadric (see e.g. [9]). In [8] the author studies, when $q \equiv 2(\bmod 3)$, the intersection of the unitary ovoid of $\mathcal{Q}$ with singular hyperplanes which are polar hyperplanes, with respect to the polarity defined by $\mathcal{Q}$, of points not belonging to the ovoid. These intersections project into ovoids of $\mathcal{Q}^{+}(5, q)$. When $q \equiv 0(\bmod 3)$, he considers the intersection of the unitary ovoid of $\mathcal{Q}^{\prime}$ both with hyperplanes which are polar hyperplanes of singular points not belonging to the ovoid and with non-singular hyperplanes intersecting $\mathcal{Q}^{\prime}$ in a hyperbolic quadric $\mathcal{Q}^{+}(5, q)$, obtaining ovoids of $\mathcal{Q}(4, q)$ and $\mathcal{Q}^{+}(5, q)$, respectively. These ovoids produce, through the Klein correspondence, spreads of $\mathrm{PG}(3, q)$ and hence translation planes of order $q^{2}$.

Regarding the unitary spread in [9] the author exhibits three slices inequivalent under the action of $\operatorname{PGU}(3, q)$, and hence three inequivalent symplectic 2-spreads of $\operatorname{PG}(5, q)$.

A line spread (a spread for short) $\mathcal{S}$ of $\operatorname{PG}(5, q)$ is a set of lines partitioning the point set of $\operatorname{PG}(5, q)$. The spread $\mathcal{S}$ is said to be normal if it induces a spread in any 3 -dimensional subspace of $\operatorname{PG}(5, q)$ generated by two of its elements. In [11], the author introduces an isomorphism $\beta$ between the classical unital of the desarguesian projective plane $\mathrm{PG}\left(2, q^{2}\right)$ and the unitary ovoid introduced by Kantor, by means of the Grassmannian variety $\mathcal{G}$ of the lines of a 5-dimensional projective space (for definitions and details on the Grassmannian variety see e.g. [7]). Precisely, it is proven that a normal spread of $\operatorname{PG}(5, q)$ is represented on the Grassmannian of the subspaces of rank 2 of $\operatorname{PG}(5, q)$ by a cap, say $\mathcal{V}$, of $\operatorname{PG}(8, q)$. A Hermitian curve of $\mathrm{PG}\left(2, q^{2}\right)$ is represented by a hyperplane section of $\mathcal{V}$ and, for $q \equiv 0,2(\bmod 3)$, this section is contained in the hyperbolic quadric $\mathcal{Q}$ and it is isomorphic, through $\beta$, to the unitary ovoid [11, Theorem 6] (see also [2]). In this article, using the isomorphism $\beta$ and the classification of the intersection set of a pencil of Hermitian curves in $\mathrm{PG}\left(2, q^{2}\right)$ obtained by Kestenband in [10], we prove that the slices of the unitary spread of $\mathcal{Q}$, for $q \equiv 2(\bmod 3)$, can be partitioned into five disjoint classes according to the geometric structure of the intersection between the non-singular hyperplane and the unitary ovoid of $\mathcal{Q}$. Hyperplanes belonging to different classes are non-equivalent under the action of the stabilizer of the unitary spread in the orthogonal group $P \Gamma O^{+}(8, q)$. When $q$ is even (i.e. $q=2^{2 h+1}$ ), this provides the determination of all possible inequivalent symplectic spreads of $\operatorname{PG}(5, q)$ arising from the unitary spread of $\mathcal{Q}^{+}\left(7,2^{2 h+1}\right)$, via the construction of Dillon and Dye.

Finally, we use a same approach to study the intersections of the unitary spread of $\mathcal{Q}^{\prime}=\mathcal{Q}(6, q), q \equiv 0(\bmod 3)$ with hyperplanes of $\mathrm{PG}(6, q)$. Precisely, we focus 
on the slices of the unitary spread of $\mathcal{Q}^{\prime}$ with respect to non-singular hyperplanes intersecting $\mathcal{Q}^{\prime}$ in an elliptic quadric $\mathcal{Q}^{-}(5, q)$. These hyperplanes are divided into two orbits under the action of the group stabilizing the unitary spread of $\mathcal{Q}^{\prime}$. Also in this case, hyperplanes belonging to these orbits are characterized in terms of the geometric structure of their intersection with the unitary ovoid $\Omega^{\prime}$ of $\mathcal{Q}^{\prime}$. Intersecting any element of the unitary spread with such hyperplanes we obtain two classes of line spreads of $Q^{-}(5, q)$ and hence two classes of ovoids of the Hermitian surface $\mathcal{H}\left(3, q^{2}\right)$.

\section{The setting}

We start by recalling a construction of the unitary ovoid and of the unitary spread exhibited by Kantor in [8].

Denote by $\mathbb{M}$ the 9 -dimensional vector space of all the $3 \times 3$-matrices over $\mathbb{F}_{q^{2}}$ and, for any $M \in \mathbb{M}$, set $\bar{M}=M^{q}$, also denote by $M^{t}$ the transpose of an element of $\mathbb{M}$. Consider the following $\mathbb{F}_{q}$-vector subspace of $\mathbb{M}$

$$
\mathbb{V}=\left\{\left(\begin{array}{ccc}
x & y & c \\
z & a & y^{q} \\
b & z^{q} & x^{q}
\end{array}\right): x, y, z \in \mathbb{F}_{q^{2}}, a, b, c \in \mathbb{F}_{q} \text { and } a+x+x^{q}=0\right\}
$$

Thus, $\mathbb{V}$ is an 8 -dimensional $\mathbb{F}_{q}$-vector subspace of $\mathbb{M}$. Let $\mathbb{P}=\operatorname{PG}(7, q)$ be the projective space associated with $\mathbb{V}$, i.e. the lattice of all vector subspaces of $\mathbb{V}$ and let

$$
Q(M)=\operatorname{Tr}(x)^{2}-N(x)+\operatorname{Tr}(y z)+b c,
$$

where $\operatorname{Tr}: x \in \mathbb{F}_{q^{2}} \mapsto x+x^{q} \in \mathbb{F}_{q}$ and $N: x \in \mathbb{F}_{q^{2}} \mapsto x^{q+1} \in \mathbb{F}_{q}$. Then, $Q(M)=0$ is a quadric of $\mathbb{P}$ with associated bilinear form $Q(M+N)-Q(M)-Q(N)=\operatorname{tr}(M N)$. Now, $Q(M)=0$ is a hyperbolic quadric $\mathcal{Q}=\mathcal{Q}^{+}(7, q)$ of $\operatorname{PG}(7, q)$ if and only if $q \equiv 2(\bmod 3)$. Moreover, if $q=3^{h}$, the quadric $Q(M)=0$ is a cone, say $\mathcal{C}$, of $\mathbb{P}$ with vertex $\langle I\rangle$, where $I$ is the identity matrix, having as a base the parabolic quadric $\mathcal{Q}(6, q)$ (see [9]). If $q \equiv 2(\bmod 3)$ the set $\Omega=\left\{\langle X\rangle \in \mathbb{V} \mid X^{2}=0\right\}$ consists of $q^{3}+1$ points of $\mathcal{Q}$ pairwise non-perpendicular, that is, $\Omega$ is an ovoid of $\mathcal{Q}$, while, if $q=3^{h}$, it projects into an ovoid, say $\Omega^{\prime}$, of the non-singular parabolic quadric $\mathcal{Q}(6, q)$. Precisely, $\Omega$ consists of the points

$$
\left(\begin{array}{lll}
0 & 0 & 1 \\
0 & 0 & 0 \\
0 & 0 & 0
\end{array}\right) \text { and }\left(\begin{array}{ccc}
\alpha & \alpha \beta^{q} & \alpha^{q+1} \\
\beta & \beta^{q+1} & \alpha^{q} \beta \\
1 & \beta^{q} & \alpha^{q}
\end{array}\right)
$$

with $\alpha, \beta \in \mathbb{F}_{q^{2}}$ such that $\operatorname{Tr}(\alpha)+N(\beta)=0$. While $\Omega^{\prime}$ consists of the points

$$
\left(\begin{array}{lll}
0 & 0 & 1 \\
0 & 0 & 0 \\
0 & 0 & 0
\end{array}\right) \text { and }\left(\begin{array}{ccc}
\alpha+\operatorname{Tr}(\alpha) & \alpha \beta^{q} & \alpha^{q+1} \\
\beta & 0 & \alpha^{q} \beta \\
1 & \beta^{q} & \alpha^{q}+\operatorname{Tr}(\alpha)
\end{array}\right)
$$


with $\alpha, \beta \in \mathbb{F}_{q^{2}}$ such that $\operatorname{Tr}(\alpha)+N(\beta)=0$. Here the parabolic quadric $\mathcal{Q}(6, q)$ containing $\Omega^{\prime}$ has equation

$$
x^{2}+\operatorname{Tr}(y z)+b c=0
$$

i.e. $\mathcal{Q}(6, q)=\Pi \cap \mathcal{C}$ where $\Pi$ is the hyperplane of $\mathbb{P}$ with equation $\operatorname{Tr}(x)=0$. The ovoids $\Omega$ and $\Omega^{\prime}$ are called the unitary ovoids of $\mathcal{Q}$ and $\mathcal{Q}(6, q)$, respectively.

Finally, observe that, if $q \equiv 1(\bmod 3)$, then the quadratic form $Q(M)$ defines in $\operatorname{PG}(7, q)=\operatorname{PG}\left(\mathbb{V}, \mathbb{F}_{q}\right)$ an elliptic quadric $\mathcal{Q}^{-}(7, q)$. In this case, the set $\Omega=\{\langle X\rangle \in$ $\left.\mathbb{V} \mid X^{2}=0\right\}$ is a partial ovoid of $\mathcal{Q}^{-}(7, q)$.

Let $\mathrm{GU}(3, q)$ be the unitary group of all the non-singular $3 \times 3$ matrices $A$ over $\mathbb{F}_{q^{2}}$ such that $J A J=\left(\bar{A}^{t}\right)^{-1}$, where

$$
J=\left(\begin{array}{lll}
0 & 0 & 1 \\
0 & 1 & 0 \\
1 & 0 & 0
\end{array}\right) .
$$

The group $\mathrm{GU}(3, q)$ acts on $\mathbb{V}$ by conjugation inducing $\operatorname{PGU}(3, q)$ on $\operatorname{PG}(7, q)$; nevertheless, $\mathrm{GU}(3, q)$ preserves the quadric $Q(M)=0$ and acts 2-transitively on the set $\Omega([9])$.

Let $T(X)=\{M \in \mathbb{V}: X M=M X=0\}$, where $X$ is a point of $\Omega$. Then, $T(X)$ is a totally singular plane, and $T(X)$ is disjoint from $T(Y)$ if $X$ and $Y$ are distinct points of $\Omega$. If $q \equiv 2(\bmod 3)$, we can fix one type of maximal totally singular subspaces of $\mathcal{Q}$, denote by $F(X)$ the subspace of fixed type containing $T(X)$ for any matrix $X \in \Omega$, and set $\mathcal{S}_{U}=\{F(X): X \in \Omega\}$. The spread $\mathcal{S}_{U}$ is called the unitary spread of $\mathcal{Q}$.

On the other hand, if $q=3^{h}$, the projection of any $T(X)$ from the vertex $\langle I\rangle$ of $\mathcal{C}$ defines a totally singular plane, say $T(X)^{\prime}$, of $\mathcal{Q}(6, q)$. The set $\mathcal{S}=\left\{T(X)^{\prime}: X \in \Omega\right\}$ is called in [11] the unitary spread of $\mathcal{Q}(6, q)$.

Moreover, we can embed $\mathcal{Q}(6, q)$ in a hyperbolic quadric $\mathcal{Q}^{+}(7, q)$ of a $\operatorname{PG}(7, q)$ as intersection of $\mathcal{Q}^{+}(7, q)$ with a non-singular hyperplane of $\operatorname{PG}(7, q)$; the set $\Omega^{\prime}$ is an ovoid of $\mathcal{Q}^{+}(7, q)$ as well and $\tilde{\mathcal{S}}=\{M(X), X \in \Omega\}$, where $M(X)$ is the totally singular 3-dimensional subspace of a fixed type of $\mathcal{Q}^{+}(7, q)$ containing $T(X)^{\prime}$, is a spread of $\mathcal{Q}^{+}(7, q)$. It will be useful for our purposes to consider this spread, too. The spread $\tilde{\mathcal{S}}$ is known as the unitary spread of $\mathcal{Q}^{+}(7, q)$ when $q=3^{h}$.

Note that unitary spread and unitary ovoid in $Q^{+}(7, q)$ correspond to each other via a triality map $\tau$ of the hyperbolic quadric $\mathcal{Q}^{+}(7, q)$. This can be extracted from [16].

\section{Preliminary results}

In this section we briefly recall the work done in [11, §4] and show some lemmas and propositions that will be useful in the proof of our theorems.

A normal spread of $\operatorname{PG}(5, q)$ can be constructed in the following way. Let $\Sigma^{*}=\mathrm{PG}\left(5, q^{2}\right)$ and let $\left(x_{0}, x_{1}, x_{2}, x_{3}, x_{4}, x_{5}\right)$ be the projective homogeneous coordinates of a point of $\Sigma^{*}$. Denote by $\sigma$ the involutory collineation of $\Sigma^{*}$ defined 
by $\left(x_{0}, x_{1}, x_{2}, x_{3}, x_{4}, x_{5}\right)^{\sigma}=\left(x_{3}^{q}, x_{4}^{q}, x_{5}^{q}, x_{0}^{q}, x_{1}^{q}, x_{2}^{q}\right)$. The set of points fixed by $\sigma$ is a canonical subgeometry of $\Sigma^{*}$, i.e. $\Sigma=\left\{\left(x_{0}, x_{1}, x_{2}, x_{0}^{q}, x_{1}^{q}, x_{2}^{q}\right): x_{0}, x_{1}, x_{2} \in \mathbb{F}_{q^{2}}\right\}$. Let $\pi \subset \Sigma^{*}$ be a plane with equations $x_{3}=x_{4}=x_{5}=0$. Then $\pi$ is disjoint from $\Sigma$ and the plane $\pi^{\sigma}$ has equations $x_{0}=x_{1}=x_{2}=0$. For each point $x$ of $\pi$, let $L(x)=\left\langle x, x^{\sigma}\right\rangle$, be the line joining the points $x$ and $x^{\sigma}$ and put $\mathcal{S}^{*}=\{L(x): x \in \pi\}$. Then, $\mathcal{S}=\{L(x) \cap \Sigma: x \in \pi\}$ is a line spread of $\Sigma$ which turns out to be a normal spread. It is easy to show that the Grassmannian map $g$ from the lines of $\Sigma^{*}$ into the points of $\Lambda^{*}=\operatorname{PG}\left(14, q^{2}\right)$ maps the set $\mathcal{S}^{*}=\{L(x): x \in \pi\}$ into an 8 -dimensional projective subspace $\Delta^{*}$ of $\Lambda^{*}$. Precisely $\Delta^{*}$ has equations $p_{01}=$ $p_{02}=p_{12}=p_{34}=p_{35}=p_{45}=0$, and any of its point has homogenous coordinates $\left(p_{03}, p_{04}, p_{05}, p_{13}, p_{14}, p_{15}, p_{23}, p_{24}, p_{25}\right)$.

Now, let $\mathcal{V}=g(\mathcal{S})$, i.e. let $\mathcal{V}$ be the representation of $\mathcal{S}$ on the Grassmannian $\mathcal{G}$ of the lines of $\Sigma$; this is an algebraic variety of a canonical subgeometry $\Lambda \simeq \mathrm{PG}(14, q)$ of $\Lambda^{*}$. It is easy to show that $\Delta^{*}$ is a subspace of $\Lambda$ as well, i.e. $\Delta=\Delta^{*} \cap \Lambda$ has rank 9; precisely,

$$
\Delta:=\left\{\left(x_{0}, x_{1}, x_{2}, x_{1}^{q}, x_{4}, x_{5}, x_{2}^{q}, x_{5}^{q}, x_{8}\right), x_{0}, x_{4}, x_{8} \in \mathbb{F}_{q}, x_{1}, x_{2}, x_{5} \in \mathbb{F}_{q^{2}}\right\} .
$$

Also, in [11], it has been proven that $\mathcal{V}$ is the complete intersection of the Grassmannian $\mathcal{G}$ with $\Delta$.

Note that the vector space $\Delta$ underlies an 8-dimensional projective space containing the projective space $\mathbb{P}$ associated with $\mathbb{V}$ as a hyperplane. Moreover, a point $p$ of $\Delta$ belongs to $\mathcal{V}$ if and only if

$$
p=\left(a_{0}^{1+q}, a_{0} a_{1}^{q}, a_{0} a_{2}^{q}, a_{1} a_{0}^{q}, a_{1}^{1+q}, a_{1} a_{2}^{q}, a_{2} a_{0}^{q}, a_{2} a_{1}^{q}, a_{2}^{1+q}\right),
$$

where $a_{0}, a_{1}$ and $a_{2} \in \mathbb{F}_{q^{2}}$.

Now, let $m=\langle x, y\rangle$ be a line of $\pi, S^{*}=\langle L(x), L(y)\rangle, S=S^{*} \cap \Sigma$, and let $\mathcal{N}$ be the spread of the 3-dimensional projective space $S$ induced by $\mathcal{S}$. Then the image of $\mathcal{N}$ under $g$ is an elliptic quadric $\mathcal{Q}_{m}=\mathcal{Q}^{-}(3, q)$ which is the complete intersection of $\mathcal{V}$ with a 3 -dimensional projective subspace contained in $\Delta[11$, Theorem 1$]$. Hence, the incidence structure having as points the points of $\mathcal{V}$, as lines the quadrics $\mathcal{Q}_{m}$ contained in $\mathcal{V}$ and whose incidence is the natural one, is isomorphic to $\operatorname{PG}\left(2, q^{2}\right)$ via the isomorphism $\beta$ defined by the following rules $x \mapsto g(L(x))$ and $m \mapsto \mathcal{Q}_{m}$, where $x$ and $m$ belong to the pointset and to the lineset of $\operatorname{PG}\left(2, q^{2}\right)$, respectively. If $\mathcal{H}\left(2, q^{2}\right)$ is a non-singular Hermitian curve of $\mathrm{PG}\left(2, q^{2}\right)$ with equation $x_{0} x_{2}^{q}+$ $x_{1}^{q+1}+x_{0}^{q} x_{2}$, than the image of $\mathcal{H}\left(2, q^{2}\right)$ under $\beta$ is $\Omega=\mathcal{V} \cap \mathbb{P}$ [11, Theorem 6]. This result was also independently obtained by Cooperstein [2, Lemma 2.3].

Denote by $H$ both the stabilizer of $\Omega$ in the orthogonal group $P \Gamma O^{+}(8, q)$, $q \equiv 2(\bmod 3)$ and the stabilizer of $\Omega^{\prime}$ in $P \Gamma O(7, q), q=3^{h}$. The stabilizer of the classical unital $\mathcal{H}\left(2, q^{2}\right)$ is the group $\operatorname{PGU}(3, q) \rtimes \operatorname{Aut}\left(\mathbb{F}_{q^{2}}\right)$, induced by $\operatorname{GU}(3, q)$ and, because of the above arguments, it is isomorphic to $H$. Precisely, by using the isomorphism $\beta$, one can see that the linear part $\bar{H}$ of $H$ is isomorphic to $\operatorname{PGU}(3, q) \rtimes C_{2}$, where $C_{2}$ is the subgroup of $\operatorname{Aut}\left(\mathbb{F}_{q^{2}}\right)$ of order two.

Now, denote by $G$ both the stabilizer of $\mathcal{S}_{U}, q \equiv 2(\bmod 3)$, and the stabilizer of $\tilde{\mathcal{S}}, q=3^{h}$, in the orthogonal group associated with the relevant hyperbolic quadrics. Note that when $q \equiv 0(\bmod 3)$ the stabilizer $G_{\Pi}$ of the hyperplane $\Pi$ in $G$ coincides 
with the stabilizer of the spread $\mathcal{S}$ of $Q(6, q)=\Pi \cap \mathcal{Q}^{+}(7, q)$ in $P \Gamma O(7, q)$. Denote by $\bar{G}$ and $\overline{G_{\Pi}}$ the linear part of $G$ and $G_{\Pi}$, respectively. We have the following

Proposition 3.1 The group $\bar{G}$ is isomorphic to PGU $(3, q)$ and the group, $\overline{G_{\Pi}}$ is isomorphic to $\operatorname{PGU}(3, q) \rtimes C_{2}$ where $C_{2}$ is the subgroup of $\operatorname{Aut}\left(\mathbb{F}_{q^{2}}\right)$ of order two.

Proof The unitary ovoid and the unitary spread of $\mathcal{Q}^{+}(7, q)$ are related to each other by a triality map of $\mathcal{Q}^{+}(7, q)$. Denote by $\tau$ this map and suppose $\Omega=\mathcal{S}_{U}^{\tau^{2}}$ (or $\Omega^{\prime}=$ $\tilde{\mathcal{S}}^{\tau^{2}}$ ). This means that $\tau \bar{G} \tau^{-1}$ is a subgroup of $\bar{H}$. Also, by [9, Proposition 6.15(iii)], the groups $\bar{G}$ and $\overline{G_{\Pi}}$, both contain a subgroup isomorphic to $\operatorname{PGU}(3, q)$. This means that $\bar{G}$ and $\overline{G_{\Pi}}$ are either isomorphic to $\operatorname{PGU}(3, q)$ or to $\operatorname{PGU}(3, q) \rtimes C_{2}$. Now, when $q \equiv 0(\bmod 3)$, consider

$$
X=\left(\begin{array}{lll}
0 & 0 & 1 \\
0 & 0 & 0 \\
0 & 0 & 0
\end{array}\right)
$$

then

$$
T(X)^{\prime}=\left\{\left(\begin{array}{ccc}
0 & y & c \\
0 & 0 & y^{q} \\
0 & 0 & 0
\end{array}\right) \mid y \in \mathbb{F}_{q^{2}}, c \in \mathbb{F}_{q}\right\}
$$

The group $C_{2}$ fixes $T(X)^{\prime}$. Nevertheless, suppose $q \equiv 2(\bmod 3)$, then $q=p^{2 h+1}$ with $p$ a prime number such that $p \equiv 2(\bmod 3)$. Hence, since $\mathbb{F}_{q}$ does not contain primitive cube roots of unity, the polynomial $t^{2}+t+1$ is irreducible over $\mathbb{F}_{q}$. Let $\omega$ be a root of $t^{2}+t+1$ in $\mathbb{F}_{q^{2}}$, then any element $x \in \mathbb{F}_{q^{2}}$ can be uniquely written as $x=$ $x_{0} \omega+x_{1} \omega^{q}$, where $x_{0}, x_{1} \in \mathbb{F}_{q}$. So $T(x)=-\left(x_{0}+x_{1}\right)$ and $N(x)=x_{0}^{2}-x_{0} x_{1}+x_{1}^{2}$ and it is easy to show that the two maximal totally singular subspaces containing $T(X)=T(X)^{\prime}$ are

$$
\begin{aligned}
& F_{1}(X)=\left\{\left(\begin{array}{ccc}
x_{0} \omega & y & c \\
0 & x_{0} & y^{q} \\
0 & 0 & x_{0} \omega^{q}
\end{array}\right) \mid y \in \mathbb{F}_{q^{2}} c, x_{0} \in \mathbb{F}_{q}\right\}, \\
& F_{2}(X)=\left\{\left(\begin{array}{ccc}
x_{1} \omega^{q} & y & c \\
0 & x_{1} & y^{q} \\
0 & 0 & x_{1} \omega
\end{array}\right) \mid y \in \mathbb{F}_{q^{2}} c, x_{1} \in \mathbb{F}_{q}\right\} .
\end{aligned}
$$

These are mapped one into the other by $C_{2}$. Since, up to isomorphisms, $\operatorname{PGU}(3, q)$ acts transitively on the elements $\mathcal{S}_{U}$, on the elements of the spread $\mathcal{S}$ of $Q(6, q)$ and it is normal in $\operatorname{PGU}(3, q) \rtimes C_{2}$, the assertion follows.

The next proposition can be extracted from [11] in fact, it slightly generalizes Theorem 4 of that paper:

Proposition 3.2 Any Hermitian curve (possibly singular) of $\mathrm{PG}\left(2, q^{2}\right)$ is isomorphic, via $\beta$, to the intersection $W \cap \mathcal{V}$, where $W$ is a hyperplane of $\Delta$.

Hence, we have the following 
Proposition 3.3 Let $K$ be any hyperplane of $\mathbb{P}$. Then the intersection $K \cap \Omega$ is isomorphic to the intersection set of a pencil of Hermitian curves of $\mathrm{PG}\left(2, q^{2}\right)$, one of them being $\mathcal{H}\left(2, q^{2}\right)$.

Proof Recall that, by [11], $\Omega=\mathbb{P} \cap \mathcal{V}$. Since $K$ is a 6-dimensional subspace of $\Delta$, $K=W_{1} \cap W_{2} \cap \cdots \cap W_{q+1}$, where $W_{i}, i=1, \ldots, q+1$, is a hyperplane of $\Delta$ and we can put $W_{1}=\mathbb{P}$. Hence, we have $K \cap \Omega=W_{1} \cap W_{2} \cap \cdots \cap W_{q+1} \cap \Omega=W_{1} \cap W_{2} \cap$ .. $\cap W_{q+1} \cap \mathcal{V}$. By Proposition 3.2, $K \cap \Omega$ is then isomorphic to the intersection set of a pencil of $q+1$ Hermitian curves of $\mathrm{PG}\left(2, q^{2}\right)$ and $\mathbb{P} \cap \mathcal{V}$ corresponds to $\mathcal{H}\left(2, q^{2}\right)$.

Lemma 3.4 A collineation $h \in H$ fixes a hyperplane $K$ of $\mathbb{P}$ (a hyperplane $U$ of $\Pi$ ) if and only if h fixes the intersection $K \cap \Omega$ (the intersection $U \cap \Omega^{\prime}$ ).

Proof We only need to prove the sufficient condition. To this purpose let $h$ be a collineation of $H$ fixing $K \cap \Omega$ and suppose $K \neq K^{h}$. Then,

$$
K=W_{1} \cap W_{2} \cap \cdots \cap W_{q+1} \quad \text { and } \quad K^{h}=W_{1}^{\prime} \cap W_{2}^{\prime} \cap \cdots \cap W_{q+1}^{\prime} \text {, }
$$

where $W_{i}$ and $W_{i}^{\prime}, i=1, \ldots, q+1$, are the hyperplanes of $\Delta=\mathrm{PG}(8, q)$ containing $K$ and $K^{h}$, respectively. We can suppose $W_{1}=W_{1}^{\prime}=\mathbb{P}$. Now, by Proposition 3.2 each $W_{i}$ and $W_{i}^{\prime}, i=1, \ldots, q+1$, corresponds, via the isomorphism $\beta$, to a Hermitian curve (possibly degenerate) of $\mathrm{PG}\left(2, q^{2}\right)$; moreover both sets of $q+1$ Hermitian curves define a pencil in $\operatorname{PG}\left(2, q^{2}\right)$, whose base is $(K \cap \Omega)^{\beta^{-1}}$. Since $K \cap \Omega=K^{h} \cap \Omega$, there exist two pencils of Hermitian curves both containing the curve $\mathcal{H}\left(2, q^{2}\right)$ with the same base; a contradiction. Hence, if $h$ fixes $K \cap \Omega$, then $h$ fixes $K$. The same holds if we start by considering $h$ fixing $U \cap \Omega^{\prime}$ were $U$ is a hyperplane of $\Pi=\operatorname{PG}(6, q)$; indeed, it is enough to observe that any such 5dimensional projective space can be uniquely extended to a hyperplane, say $K$ of $\mathbb{P}$ passing through the vertex $\langle I\rangle$ of the cone $\mathcal{C}$ and that the group $H$ fixes the vertex $\langle I\rangle$ of the cone.

Now, put $\mathcal{H}_{1}=\mathcal{H}\left(2, q^{2}\right)=\Omega^{\beta^{-1}}$ and denote by $\mathcal{H}_{2}$ any other Hermitian curve of $\mathrm{PG}\left(2, q^{2}\right)$, possibly singular. So, $\mathcal{H}_{2}$ can be one of the following types: non-singular, a Hermitian cone with vertex $V$ and finally a line repeated $q+1$ times. Also, denote by $\mathcal{E}=\mathcal{H}_{1} \cap \mathcal{H}_{2}$, the intersection of $\mathcal{H}_{1}$ and $\mathcal{H}_{2}$, and by $|\mathcal{E}|$ the size of $\mathcal{E}$. The set $\mathcal{E}$ defines a pencil of $q+1$ Hermitian curves of $\operatorname{PG}\left(2, q^{2}\right)$ which is independent of the choice of $\mathcal{H}_{1}$ and $\mathcal{H}_{2}$ in the pencil.

In [10], the author completely classified all possibilities for the set $\mathcal{E}$ and the geometric configurations of $\mathcal{E}$ can be extracted from Table 1 .

Using this classification and Propositions 3.3 and 3.2, we have that $1,(q+1)^{2}$, $q^{2}+q+1, q^{2}+1, q+1$, and $q^{2}-q+1$ are also the possible sizes for the intersection of $\Omega$ with a hyperplane of $\mathbb{P}$.

In what follows we will denote by $\mathcal{E}_{i}, i \in\{\mathrm{I}, \mathrm{II}, \ldots, \mathrm{VII}\}$, the intersection set whose geometric structure ensues from lines I, II, ..., VII listed in Table 1, respectively. 
Table 1

\begin{tabular}{lll}
\hline & $|\varepsilon|$ & $\mathcal{H}_{2}$ \\
\hline I & $(q+1)^{2}$ & $\begin{array}{l}\text { Hermitian cone; } V \notin \mathcal{H}_{1} ; \\
\text { the lines are chords of } \mathcal{H}_{1}\end{array}$ \\
II & $q^{2}+q+1$ & $\begin{array}{l}\text { Hermitian cone; } V \in \mathcal{H}_{1} ; \\
\text { the lines are chords of } \mathcal{H}_{1}\end{array}$ \\
III & $q^{2}+1$ & $\begin{array}{l}\text { Hermitian cone; } V \notin \mathcal{H}_{1} ; \\
\text { 2 tangents and } q-1 \text { chords of } \mathcal{H}_{1}\end{array}$ \\
IV & $q^{2}+1$ & $\begin{array}{l}\text { Hermitian cone; } V \in \mathcal{H}_{1} ; \\
\text { one tangent and } q \text { chords of } \mathcal{H}_{1}\end{array}$ \\
V & $q+1$ & $\begin{array}{l}\text { a chord of } \mathcal{H}_{1} \\
\text { a tangent line of } \mathcal{H}_{1}\end{array}$ \\
VI & 1 & non-singular Hermitian curve \\
VII & $q^{2}-q+1$ & \\
\hline & &
\end{tabular}

Lemma 3.5 Consider the non-singular Hermitian curve $\mathcal{H}_{1}$ of $\mathrm{PG}\left(2, q^{2}\right)$. We have that:

1. there are $\frac{q^{3}\left(q^{2}-q+1\right)(q-1)(q-2)}{6}$ sets of type $\mathcal{E}_{\mathrm{I}}$;

2. there are $q^{2}\left(q^{3}+1\right)(q-1)$ sets of type $\mathcal{E}_{\mathrm{II}}$;

3. there are $\frac{q^{4}\left(q^{3}+1\right)}{2}$ sets of type $\mathcal{E}_{\mathrm{III}}$;

4. there are $q\left(q^{3}+1\right)(q+1)$ sets of type $\mathcal{E}_{\mathrm{IV}}$;

5. there are $q^{2}\left(q^{2}-q+1\right)$ sets of type $\mathcal{E}_{\mathrm{V}}$;

6. there are $q^{3}+1$ sets of type $\mathcal{E}_{\mathrm{VI}}$;

7. there are $\frac{q^{3}(q+1)^{3}(q-1)}{3}$ sets of type $\mathcal{E}_{\mathrm{VII}}$.

Proof The number of distinct intersection sets $\mathcal{E}_{\mathrm{V}}$ equals the number of chords of $\mathcal{H}_{1}$, while the number of distinct intersection sets $\mathcal{E}_{\mathrm{VI}}$ equals the number of points of $\mathcal{H}_{1}$. These can be easily computed, proving points 5 and 6 , respectively. Since the pencils with intersection sets $\mathcal{E}_{\mathrm{II}}, \mathcal{E}_{\mathrm{III}}$ and $\mathcal{E}_{\mathrm{IV}}$ contain exactly one cone $\mathcal{H}_{2}$ of the type described in Table 1, counting the number of these intersection sets is equivalent to counting the number of these cones. On the other hand, since there are three cones of the same type in a pencil having as intersection set one of type $\mathcal{E}_{\mathrm{I}}$, the number of such intersection sets is the number of the cones described in Table 1 divided by three. Let $V$ be the vertex of the cone, if $V \in \mathcal{H}_{1}$ and $\ell$ is a line of $\operatorname{PG}\left(2, q^{2}\right)$ not through $V$, then there exists exactly one point $P \in \ell$ such that the line $\langle V, P\rangle$ is a tangent line to $\mathcal{H}_{1}$. Hence, the number of cones defining a sets $\mathcal{E}_{\mathrm{II}}$ is $\left(q^{3}+1\right) N_{1}$ and the number of cones defining intersection sets $\mathcal{E}_{\mathrm{IV}}$ is $\left(q^{3}+1\right) N_{2}$, where $N_{1}$ and $N_{2}$ are the number of Baer sublines of $\ell$ not through $P$ and the number of Baer sublines of $\ell$ through $P$, respectively. On the other hand, if $V \notin \mathcal{H}_{1}$, then there exists a Baer subline of $\ell$, say $\ell^{\prime}$, such that the lines joining $V$ with any of the points of $\ell^{\prime}$ are tangent, the others being chords. Hence, the number of cones defining intersection sets $\mathcal{E}_{\mathrm{I}}$ is $q^{2}\left(q^{2}-q+1\right) N_{3} / 3$ and the number of cones defining intersection sets $\mathcal{E}_{\text {III }}$ is $q^{2}\left(q^{2}-q+1\right) N_{4}$, where $N_{3}$ is the number of Baer sublines of $\ell$ skew to $\ell^{\prime}$ and $N_{4}$ is the number of Baer sublines of $\ell$ having two points in common with $\ell^{\prime}$. Finally, the numbers $N_{i}, i=1, \ldots, 4$, can be easily computed using the isomorphism between 
the projective line PG $\left(1, q^{2}\right)$ and the elliptic quadric $\mathcal{Q}^{-}(3, q)$ (see [6], Chap. 15). As a consequence of Proposition 3.3, we get that the number of remaining intersection sets, i.e., intersection sets $\mathcal{E}_{\mathrm{VII}}$, is $\frac{q^{3}(q+1)^{3}(q-1)}{3}$. This concludes the proof.

Now we will determine the subgroup of the unitary group $\operatorname{PGU}(3, q)$ associated with the non-singular Hermitian curve $\mathcal{H}\left(2, q^{2}\right)$, fixing each $\mathcal{E}_{i}, i \in\{\mathrm{I}, \mathrm{II}, \ldots, \mathrm{VII}\}$. In what follows we will denote by $\mathbb{Z}_{h}$ a cyclic group of order $h$. The linear automorphism group $\operatorname{Aut}\left(\mathcal{E}_{i}\right)$ (i.e. the subgroup of $\operatorname{PGL}\left(3, q^{2}\right)$ fixing $\left.\mathcal{E}_{i}\right)$ has been computed in [5], for all $i \in\{\mathrm{I}, \mathrm{II}, \ldots, \mathrm{VII}\}$. It is easy to see that, up to isomorphism, $\operatorname{Aut}\left(\mathcal{E}_{i}\right) \leq$ $\operatorname{PGU}(3, q)$ whenever $i \in\{\mathrm{III}, \mathrm{IV}, \mathrm{VII}\}$ and we have that $\operatorname{Aut}\left(\mathcal{E}_{\mathrm{III}}\right) \simeq \mathbb{Z}_{2} \rtimes \mathbb{Z}_{q^{2}-1}$, $\operatorname{Aut}\left(\mathcal{E}_{\mathrm{IV}}\right) \simeq E_{q} \times \operatorname{AGL}(1, q)$, where $E_{q}$ is an elementary abelian group of order $q$, and finally $\operatorname{Aut}\left(\mathcal{E}_{\mathrm{VII}}\right) \simeq \mathbb{Z}_{3} \rtimes \mathbb{Z}_{q^{2}-q+1}$. By [5, Lemma 2.6], $\operatorname{Aut}\left(\mathcal{E}_{\mathrm{II}}\right) \cap \operatorname{PGU}(3, q) \simeq$ $E_{q} \rtimes \mathbb{Z}_{q+1}$. Also, $\operatorname{Aut}\left(\mathcal{E}_{\mathrm{V}}\right)$ is the subgroup of $\operatorname{PGU}(3, q)$ fixing a chord of $\mathcal{H}_{1}$, and $\operatorname{Aut}\left(\mathcal{E}_{\mathrm{VI}}\right)$ is the subgroup of $\operatorname{PGU}(3, q)$ fixing a point of $\mathcal{H}_{1}$. Finally, regarding the stabilizer of $\mathcal{E}_{\mathrm{I}}$ in $\operatorname{PGU}(3, q)$, we have the following result.

Proposition 3.6 Denote by $E$ the group $\operatorname{Aut}\left(\mathcal{E}_{\mathrm{I}}\right) \cap \operatorname{PGU}(3, q)$. Then, we have the following possibilities:

1. if $q=2^{2 h}$, then either $E \simeq\left(\mathbb{Z}_{q+1} \times \mathbb{Z}_{q+1}\right) \rtimes \mathbb{Z}_{3}$ or $E \simeq \mathbb{Z}_{q+1} \times \mathbb{Z}_{q+1}$;

2. if $q=2^{2 h+1}$, then $E \simeq \mathbb{Z}_{q+1} \times \mathbb{Z}_{q+1}$;

3. if $q=3^{h}$, then either $E \simeq\left(\mathbb{Z}_{q+1} \times \mathbb{Z}_{q+1}\right) \rtimes \operatorname{Sym}_{3}$ or $E \simeq \mathbb{Z}_{q+1} \times \mathbb{Z}_{q+1}$;

4. if $q=p^{h}$ and $p \neq 3,2$, then either $E \simeq \mathbb{Z}_{q+1} \times \mathbb{Z}_{q+1}$ or $E \simeq\left(\mathbb{Z}_{q+1} \times \mathbb{Z}_{q+1}\right) \rtimes \mathbb{Z}_{3}$, or $E \simeq\left(\mathbb{Z}_{q+1} \times \mathbb{Z}_{q+1}\right) \rtimes \mathbb{Z}_{2}$.

Proof In [5], the author reconstructs the intersection set $\mathcal{E}$ with geometric structure described in $I$ of Table 1, using as fixed non-singular Hermitian curve $\mathcal{H}$ of $\operatorname{PG}\left(2, q^{2}\right)$ containing $\mathcal{E}$, that with equation $X_{0}^{q+1}+X_{1}^{q+1}+X_{2}^{q+1}=0$. He proves that the group $\operatorname{Aut}(\mathcal{E})$ is isomorphic to $\left(\mathbb{Z}_{q+1} \times \mathbb{Z}_{q+1}\right) \rtimes \mathrm{Sym}_{3}$, where $\mathrm{Sym}_{3}$ is the symmetric group acting on three elements. It is easy to see that the unitary group associated with $\mathcal{H}$ contains the subgroup of $\operatorname{Aut}(\mathcal{E})$ isomorphic to $\mathbb{Z}_{q+1} \times \mathbb{Z}_{q+1}$. Now, the subgroup isomorphic to $\mathrm{Sym}_{3}$ is generated by the following collineations of $\operatorname{PG}\left(2, q^{2}\right)$

$$
\begin{gathered}
\sigma_{1}:\left(X_{0}, X_{1}, X_{2}\right) \mapsto\left(X_{2}, a X_{0}, b X_{1}\right), \\
\sigma_{2}:\left(X_{0}, X_{1}, X_{2}\right) \mapsto\left(c X_{1}, c^{-1} X_{0}, X_{2}\right),
\end{gathered}
$$

where $a, b, c \in \mathbb{F} q^{2}$ such that $a^{q+1}=\lambda(1-\lambda), b^{q+1}=-\frac{(1-\lambda)^{2}}{\lambda}, c^{q+1}=-\frac{1}{\lambda}$. Here $\lambda$ is an element of $\mathbb{F}_{q} \backslash\{0,1\}$ such that the Hermitian cones $\mathcal{K}_{i}, i=1,2,3$ with equations

$$
\begin{gathered}
\mathcal{K}_{1}: \lambda X_{0}^{q+1}+X_{1}^{q+1}=0, \quad \mathcal{K}_{2}:(\lambda-1) X_{1}^{q+1}+\lambda X_{2}^{q+1}=0, \\
\mathcal{K}_{3}:(1-\lambda) X_{1}^{q+1}+X_{2}^{q+1}=0
\end{gathered}
$$

belong to the pencil with base $\mathcal{E}_{\mathrm{I}}$. Hence $\operatorname{Sym}_{3} \simeq\left\{1, \sigma_{1}, \sigma_{1}^{2}, \sigma_{2}, \sigma_{3}, \sigma_{4}\right\}$, where

$$
\begin{gathered}
\sigma_{3}:\left(X_{0}, X_{1}, X_{2}\right) \mapsto\left(c a X_{0}, c^{-1} X_{2}, b X_{1}\right) \quad \text { and } \\
\sigma_{4}:\left(X_{0}, X_{1}, X_{2}\right) \mapsto\left(X_{2}, a c X_{1}, b c^{-1} X_{0}\right) .
\end{gathered}
$$


Now, the collineation $\sigma_{1}$ fixes $\mathcal{H}$ if and only if $\lambda(1-\lambda)=-\frac{(1-\lambda)^{2}}{\lambda}=1$, that is if and only if $\lambda^{2}-\lambda+1=0$. Moreover, $\sigma_{2}$ fixes $\mathcal{H}$ if and only if $\lambda=-1$. Nevertheless, $\sigma_{3}$ fixes $\mathcal{H}$ if and only if $2 \lambda=1$ and finally, $\sigma_{4}$ fixes $\mathcal{H}$ if and only if $\lambda=2$. If $q$ is even, then $\sigma_{2}, \sigma_{3}$ and $\sigma_{4} \notin E$. Moreover, if $q=2^{2 h+1}$, then $\sigma_{1} \notin E$ as well; while if $q=2^{2 h}$, then $\sigma_{1} \in E$ if and only if $\lambda^{2}-\lambda+1=0$. This proves points 1 and 2 . If $q=p^{h}$ and $p \neq 3,2$, then there are three possibilities, precisely, either $\lambda$ is an element of $\mathbb{F}_{q}$ such that $\lambda^{2}-\lambda+1=0$ or $\lambda \in\left\{-1,2, \frac{1}{2}\right\}$ or $\lambda^{2}-\lambda+1 \neq 0$ and $\lambda \notin\left\{-1,2, \frac{1}{2}\right\}$. As a consequence, we have the three stated forms for the group $E$. Finally, if $q=3^{h}$ then $E$ has the described form according whether $\lambda=-1$ or $\lambda \neq-1$.

\section{Slices of the unitary spread}

Let $q \equiv 2(\bmod 3)$ and let $\mathcal{S}_{U}$ and $\Omega$ be the unitary spread and the unitary ovoid of the hyperbolic quadric $\mathcal{Q}=\mathcal{Q}^{+}(7, q)$ of $\mathbb{P}$ defined by the Quadratic form (1), respectively. Let $K$ be a non-singular hyperplane of $\mathbb{P}$; the slice of $\mathcal{S}_{U}$ with respect to $K$ is the 2-spread induced by $\mathcal{S}_{U}$ in the parabolic quadric obtained intersecting $\mathcal{Q}$ with $K$. Note that the stabilizer $G_{K}$ of $K$ in $G$ coincides with the stabilizer, in the orthogonal group associated with the parabolic quadric, of the slice determined by $K$. As observed in the previous section, any hyperplane of $\mathbb{P}$ intersects $\Omega$ in a set of points isomorphic, via the map $\beta$, to a set $\mathcal{E}_{i}$, where $i$ varies in the set $\{$ I, II, III, IV, V, VI, VII $\}$. We say that a hyperplane $K$ of $\mathbb{P}$, is of type $i$ for $i \in\{\mathrm{I}, \mathrm{II}, \mathrm{III}, \mathrm{IV}, \mathrm{V}, \mathrm{VI}, \mathrm{VII}\}$, if $(\Omega \cap K)^{\beta^{-1}}=\mathcal{E}_{i}$. We prove the following

Proposition 4.1 Let $\mathcal{Q}=\mathcal{Q}^{+}(7, q), q \equiv 2(\bmod 3)$; there are five disjoint classes of slices of the unitary spread $\mathcal{S}_{U} \subset \mathcal{Q}$. These are obtained intersecting $\mathcal{S}_{U}$ with hyperplanes of $\mathbb{P}$ of types $i$, where $i \in\{\mathrm{I}, \mathrm{II}, \mathrm{III}, \mathrm{V}, \mathrm{VII}\}$. Slices obtained by intersecting $\mathcal{Q}$ with hyperplanes of different types are not equivalent under the action of the group $G$.

Proof By Proposition 3.1, we have that the linear part $\bar{G}$ of $G$ is isomorphic to the unitary group PGU $(3, q)$. Let $K$ be any hyperplane of $\mathbb{P}$. By Lemma 3.4, the stabilizer in the group $\bar{G}$ of $K$, i.e. the linear stabilizer of the slice determined by $K$, coincides with the stabilizer in $\operatorname{PGU}(3, q)$ of $K \cap \Omega$ and by Proposition 3.2, it is isomorphic to the stabilizer in the relevant projective unitary group of one of the intersection sets $\mathcal{E}_{i}, i \in\{\mathrm{I}, \mathrm{II}, \ldots, \mathrm{VI}, \mathrm{VII}\}$. These groups and their orders have been described and discussed in Sect. 2. In what follows we will determine which intersection sets $\mathcal{E}_{i}$, $i \in\{\mathrm{I}, \mathrm{II}, \mathrm{III}, \mathrm{IV}, \mathrm{V}, \mathrm{VI}, \mathrm{VII}\}$, correspond, through the map $\beta$, to the intersection of $\Omega$ with non-singular hyperplanes of $\mathbb{P}$. To this aim, we first observe that if $K$ is a singular hyperplane polar of a point $P \in \Omega$, then $K \cap \Omega=P$. Hence, the hyperplane $K$ corresponds, via the isomorphism $\beta$, to an intersection set $\mathcal{E}_{\mathrm{VI}}$. This provides an orbit of such hyperplanes of length $q^{3}+1$ under the action of $G$. On the other hand, if $K$ is a singular hyperplane polar of a point $P \notin \Omega$, then $K \cap \Omega$ projects into an ovoid of a $\mathcal{Q}^{+}(5, q)$ [8]. So, $|K \cap \Omega|=q^{2}+1$. There are two types of intersection sets of this size namely, the $\mathcal{E}_{\text {III }}$ 's and the $\mathcal{E}_{\text {IV }}$ 's, (see Table 1 ). Now, let

$$
P=\left(\begin{array}{lll}
0 & 1 & 0 \\
0 & 0 & 1 \\
0 & 0 & 0
\end{array}\right) ;
$$


then $P \in \mathcal{Q}^{+}(7, q) \backslash \Omega$ and $K=P^{\perp}$ has equation $\operatorname{Tr}(z)=0$. So, we have

$$
K \cap \Omega=\left\{\left(\begin{array}{ccc}
0 & 0 & 1 \\
0 & 0 & 0 \\
0 & 0 & 0
\end{array}\right),\left(\begin{array}{ccc}
\alpha & \alpha \beta^{q} & \alpha^{q+1} \\
\beta & \beta^{q+1} & \alpha^{q} \beta \\
1 & \beta^{q} & \alpha^{q}
\end{array}\right) \mid \operatorname{Tr}(\beta)=0 \text { and } \operatorname{Tr}(\alpha)+N(\beta)=0\right\} .
$$

It is easy to show that $K \cap \Omega$ is isomorphic to the intersection, in $\operatorname{PG}\left(2, q^{2}\right)$, between the Hermitian curve $\mathcal{H}_{1}$ and the Hermitian cone $\mathcal{K}$ with equation $X_{1} X_{2}^{q}+X_{1}^{q} X_{2}=0$. The cone $\mathcal{K}$ has vertex $V=\langle(1,0,0)\rangle_{\mathbb{F}_{q}}$, hence $V \in \mathcal{H}_{1}$ and so the hyperplane $K=P^{\perp}$ corresponds to a subset $\mathcal{E}_{\mathrm{IV}}$ of $\mathcal{H}_{1}$. The subgroup of $\operatorname{PGU}(3, q)$ fixing such intersection has order $q^{2}(q-1)$ (see [5]), hence the orbit of $K$ under the action of this group has length $\frac{q^{3}\left(q^{3}+1\right)\left(q^{2}-1\right)}{q^{2}(q-1)}=q\left(q^{3}+1\right)(q+1)$. There are $q^{3}\left(q^{3}+1\right)$ remaining singular points; since the subgroup of $\operatorname{PGU}(3, q)$ fixing a intersection set $\mathcal{E}_{\text {III }}$ has order $2\left(q^{2}-1\right)$, the orbit of any of this point, under the action of the mentioned group, has length $\frac{q^{3}\left(q^{3}+1\right)}{2}$. So, by Lemma 3.5, we conclude that there are two orbits of singular hyperplanes and $q-2$ orbits of non-singular hyperplanes intersecting $\Omega$ in a set corresponding through $\beta$ to an intersection set of type $\mathcal{E}_{\text {III }}$ in $\mathcal{H}_{1}$. The above arguments show that non-singular hyperplanes correspond to intersection sets of type $\mathcal{E}_{i}, i \in\{\mathrm{I}, \mathrm{II}, \mathrm{III}, \mathrm{V}, \mathrm{VII}\}$ and, naturally, hyperplanes corresponding to different intersection sets are not equivalent under the action of $G$.

As mentioned in the introduction, when $q$ is even, i.e. when $q=2^{2 h+1}$ there is a connection between spreads of $\mathcal{Q}^{+}(7, q)$ and 2-spreads of $W(5, q)$.

In [8] the author exhibits three slices of $\mathcal{S}_{U} \subset \mathcal{Q}^{+}\left(7,2^{2 h+1}\right)$ non-isomorphic with respect to $\bar{G} \simeq \operatorname{PGU}(3, q)$. Precisely they are defined by the following non-singular points of $\mathbb{P}$ :

i. $N=\left(\begin{array}{lll}1 & 0 & 0 \\ 0 & 0 & 0 \\ 0 & 0 & 1\end{array}\right)$, in this case the stabilizer in $\operatorname{PGU}(3, q)$ of the corresponding slice is $\mathbb{Z}_{q+1} \times \operatorname{PGU}(2, q)$

ii. $N^{\prime}=\left(\begin{array}{ccc}a & 0 & 1 \\ 0 & 1 & 0 \\ 1 & 0 & a^{q}\end{array}\right)$ with $a \in \mathbb{F}_{q^{2}}$ such that $\operatorname{Tr}(a)=1$, in this case the stabilizer in $\operatorname{PGU}(3, q)$ of the corresponding slice is $\mathbb{Z}_{q+1} \times \mathbb{Z}_{q+1}$;

iii. all points of an anisotropic line $\ell$, in this case the slice corresponding to any point of $\ell$ has as stabilizer a cyclic group of order $q^{2}-q+1$.

The translation planes arising from these spreads are also investigated.

According to the terminology used in [9], referred to the desarguesian spread of $\mathcal{Q}^{+}(2 n+1, q)$, we say that 2-spreads of $W(5, q)$ obtained from the unitary spread $S_{U}$ of $\mathcal{Q}^{+}(7, q)$ are cousins. We are here mainly interested in those cousins that are nonequivalent under the action of the stabilizer in $P \Gamma O^{+}(8, q)$ of $S_{U}$. In what follows we will use the same symbol $\mathcal{S}$ to denote both the slices and the symplectic 2 -spreads of $\operatorname{PG}(5, q)$ they produce. Moreover, we denote by $\operatorname{Sp}(6, q) \mathcal{S}$ the stabilizer of $\mathcal{S}$ in the group $\operatorname{Sp}(6, q)$ associated with the symplectic polarity of $\operatorname{PG}(5, q)$. As a consequence of Proposition 4.1, we have the following 
Theorem 4.2 There are five classes of non-isomorphic symplectic 2-spreads of $\mathrm{PG}(5, q)$ which can be obtained from the unitary spread of $Q^{+}(7, q) q=2^{2 h+1}$; precisely

1. $\operatorname{Sp}(6, q)_{\mathcal{S}} \cong \mathbb{Z}_{q+1} \times \mathbb{Z}_{q+1}$; there are at least $\frac{q-2}{6} \frac{d}{2 h+1}$ cousins in this class, where $d$ is a divisor of $2 h+1$;

2. $\operatorname{Sp}(6, q)_{\mathcal{S}} \cong E_{q} \rtimes \mathbb{Z}_{q+1}$ where $E_{q}$ is an elementary abelian group of order $q$; there is a unique cousin in this class;

3. $\operatorname{Sp}(6, q)_{\mathcal{S}} \cong \mathbb{Z}_{2} \rtimes \mathbb{Z}_{q^{2}-1}$; there are at least $(q-2) \frac{d^{\prime}}{2 h+1}$ cousins in this class, where $d^{\prime}$ is a divisor of $2 h+1$;

4. $\operatorname{Sp}(6, q)_{\mathcal{S}} \cong \mathrm{SL}(2, q) \times \mathbb{Z}_{q+1}$; there is a unique cousin in this class;

5. $\operatorname{Sp}(6, q)_{\mathcal{S}} \cong \mathbb{Z}_{3} \rtimes \mathbb{Z}_{q^{2}-q+1}$; there are $(q+1) \frac{d^{\prime \prime}}{2 h+1}$ cousins in this class, where $d^{\prime \prime}$ is a divisor of $2 h+1$.

Proof In [8] the slices of $\mathcal{S}_{U}$ defined by the non-singular points of $\mathbb{P}$

$$
N=\left(\begin{array}{lll}
1 & 0 & 0 \\
0 & 0 & 0 \\
0 & 0 & 1
\end{array}\right) \quad \text { and } \quad N^{\prime}=\left(\begin{array}{ccc}
a & 0 & 1 \\
0 & 1 & 0 \\
1 & 0 & a^{q}
\end{array}\right) \quad \text { with } \operatorname{Tr}(a)=1
$$

have been studied. Regarding the point $N$ it is proven that the stabilizer in $\bar{G} \simeq$ $\operatorname{PGU}(3, q)$ of the corresponding slice $\mathcal{S}$ is isomorphic to $\mathbb{Z}_{q+1} \times \operatorname{SL}(2, q)$. Indeed, it is easy to show that $N^{\perp} \cap \Omega$ is isomorphic to an intersection set $\mathcal{E}_{\mathrm{V}}$. Moreover, these slices form a unique orbit under the action of the full stabilizer $G$ of $S_{U}$. Nevertheless, regarding the point $N^{\prime}$, in [8] it is proven that the stabilizer in $\bar{G}$ of the corresponding slice $\mathcal{S}$ is isomorphic to $\mathbb{Z}_{q+1} \times \mathbb{Z}_{q+1}$, indeed a straightforward calculation shows that $N^{\prime} \perp \cap \Omega$ is isomorphic to an intersection set $\mathcal{E}_{\mathrm{I}}$. These slices are partitioned into $\frac{q-2}{6}$ orbits under the action of $\bar{G}$ (see point 1 of Lemma 3.5).

Now, let

$$
N^{\prime \prime}=\left(\begin{array}{ccc}
0 & a & 1 \\
1 & 0 & a^{q} \\
0 & 1 & 0
\end{array}\right)
$$

with $a \in \mathbb{F}_{q^{2}}$ such that the polynomial $x^{3}+\operatorname{Tr}(a) x+1$ is irreducible over $\mathbb{F}_{q}$; we observe that it is always possible to choose an element in $\mathbb{F}_{q^{2}}$ with this property, in fact this is equivalent to the existence of an element $u \in \mathbb{F}_{q^{3}} \backslash \mathbb{F}_{q}$ whose trace and norm over $\mathbb{F}_{q}$ are 0 and 1 , respectively and, indeed, such an element exists for any prime power $q$ (for instance, see [12]).

The hyperplane $\left(N^{\prime \prime}\right)^{\perp}$ has equation $\operatorname{Tr}(a z)+\operatorname{Tr}(y)+b=0$. Hence,

$$
\begin{aligned}
\left(N^{\prime \prime}\right)^{\perp} & \cap \Omega \\
= & \left\{\left(\begin{array}{ccc}
\alpha & \alpha \beta^{q} & \alpha^{q+1} \\
\beta & \beta^{q+1} & \alpha^{q} \beta \\
1 & \beta^{q} & \alpha^{q}
\end{array}\right): \operatorname{Tr}\left(\alpha \beta^{q}\right)+1+\operatorname{Tr}(a \beta)=0 \text { and } \operatorname{Tr}(\alpha)+N(\beta)=0\right\} \\
& \cup\left\{\left(\begin{array}{lll}
0 & 0 & 1 \\
0 & 0 & 0 \\
0 & 0 & 0
\end{array}\right)\right\} .
\end{aligned}
$$


Since $q=2^{2 h+1}$, the polynomial $t^{2}+t+1=0$ is irreducible over $\mathbb{F}_{q}$.

Let $i \in \mathbb{F}_{q^{2}}$ such that $i^{2}+i+1=0$ and let $\left\{i, i^{q}\right\}$ be a normal basis of $\mathbb{F}_{q^{2}}$ over $\mathbb{F}_{q}$. Any element $\alpha \in \mathbb{F}_{q^{2}}$ can be uniquely written as follows $\alpha=\alpha_{1} i+\alpha_{2} i^{q}$, where $\alpha_{1}, \alpha_{2} \in \mathbb{F}_{q}$; hence we have $\operatorname{Tr}(\alpha)=\alpha_{1}+\alpha_{2}$ and $N(\alpha)=\alpha_{1}^{2}+\alpha_{2}^{2}+\alpha_{1} \alpha_{2}$. So, the system

$$
\left\{\begin{array}{l}
\operatorname{Tr}\left(\alpha \beta^{q}\right)+1+\operatorname{Tr}(a \beta)=0 \\
\operatorname{Tr}(\alpha)+N(\beta)=0
\end{array}\right.
$$

can be written as follows

$$
\left\{\begin{array}{l}
\alpha_{1} \beta_{2}+\alpha_{2} \beta_{1}+1+a \beta+a^{q} \beta^{q}=0 \\
\alpha_{1}+\alpha_{2}=N(\beta)
\end{array}\right.
$$

This system has solutions only when $\beta \in \mathbb{F}_{q^{2}} \backslash \mathbb{F}_{q}$; this implies that $\left|\left(N^{\prime \prime}\right)^{\perp} \cap \Omega\right|=$ $q^{2}-q+1$. Hence, $\left(N^{\prime \prime}\right)^{\perp} \cap \Omega$ is isomorphic to an intersection set $\mathcal{E}_{\mathrm{VII}}$ of $\mathcal{H}_{1}$. The stabilizer of the corresponding slice is then isomorphic to $\mathbb{Z}_{3} \rtimes \mathbb{Z}_{q^{2}-q+1}$. We note that the slice corresponding to the non-singular point $N^{\prime \prime}$ is one of the examples stabilized by a cyclic group of order $q^{2}-q+1$ discussed by Kantor in [9, Example 7.6]. These slices are partitioned into $q+1$ orbits under the action of $\bar{G}$ (see point 7 of Lemma 3.5).

Let

$$
N^{\prime \prime \prime}=\left(\begin{array}{ccc}
a & 0 & 1 \\
0 & 1 & 0 \\
0 & 0 & a^{q}
\end{array}\right)
$$

with $\operatorname{Tr}(a)=1$. The polar hyperplane $\left(N^{\prime \prime \prime}\right)^{\perp}$ has equation $\operatorname{Tr}\left(a^{q} x\right)+b=0$. Hence,

$$
\begin{aligned}
\left(N^{\prime \prime \prime}\right)^{\perp} \cap \Omega= & \left\{\left(\begin{array}{ccc}
\alpha & \alpha \beta^{q} & \alpha^{q+1} \\
\beta & \beta^{q+1} & \alpha^{q} \beta \\
1 & \beta^{q} & \alpha^{q}
\end{array}\right): \operatorname{Tr}\left(a^{q} \alpha\right)+1=0 \text { and } \operatorname{Tr}(\alpha)+N(\beta)=0\right\} \\
& \cup\left\{\left(\begin{array}{ccc}
0 & 0 & 1 \\
0 & 0 & 0 \\
0 & 0 & 0
\end{array}\right)\right\} .
\end{aligned}
$$

It is easy to show that $\left(N^{\prime \prime \prime}\right)^{\perp} \cap \Omega$ is isomorphic to the intersection of the Hermitian curve $\mathcal{H}_{1}$ with the Hermitian cone with equation $\operatorname{Tr}\left(a^{q} X_{0} X_{2}^{q}\right)+X_{2}^{q+1}$. Hence it corresponds to an intersection set of type $\mathcal{E}_{\text {III }}$ in $\mathcal{H}_{1}$ and the stabilizer of the corresponding slice is isomorphic to $\mathbb{Z}_{2} \rtimes \mathbb{Z}_{q^{2}-1}$. We have already shown that there are $q-2$ orbits of such non-singular hyperplanes under the action of $\bar{G}$.

Finally, let

$$
N^{\text {iv }}=\left(\begin{array}{lll}
1 & 0 & 1 \\
0 & 0 & 0 \\
0 & 0 & 1
\end{array}\right)
$$


The polar hyperplane $\left(N^{\text {iv }}\right)^{\perp}$ has equation $\operatorname{Tr}(x)+b=0$. Hence,

$$
\begin{aligned}
\left(N^{\mathrm{iv}}\right)^{\perp} \cap \Omega= & \left\{\left(\begin{array}{ccc}
\alpha & \alpha \beta^{q} & \alpha^{q+1} \\
\beta & \beta^{q+1} & \alpha^{q} \beta \\
1 & \beta^{q} & \alpha^{q}
\end{array}\right): \operatorname{Tr}(\alpha)=1 \text { and } \operatorname{Tr}(\alpha)+N(\beta)=0\right\} \\
& \cup\left\{\left(\begin{array}{lll}
0 & 0 & 1 \\
0 & 0 & 0 \\
0 & 0 & 0
\end{array}\right)\right\} .
\end{aligned}
$$

It is easy to show that $\left(N^{\text {iv }}\right)^{\perp} \cap \Omega$ is isomorphic to an intersection set $\mathcal{E}_{\text {II }}$ and the stabilizer of the corresponding slice is isomorphic to $E_{q} \rtimes \mathbb{Z}_{q+1}$. By Lemma 3.5 we have that there is a unique orbit of such non-singular hyperplanes. This concludes the proof.

We end the section with the following remark.

Remark 4.3 In [8], it is proven that the intersection of the unitary ovoid $\Omega$ with a singular hyperplane gives arise to spreads and hence to translation planes. Indeed, if $P$ is a singular point not in $\Omega$, then $P^{\perp} \cap \Omega$ projects into an ovoid of $\mathcal{Q}^{+}(5, q)$; via the Klein map, an ovoid of $\mathcal{Q}^{+}(5, q)$ corresponds to a spread of $\operatorname{PG}(3, q)$ and hence to a translation plane of order $q^{2}$. In [8] some subgroups of the automorphism group of such a spread are studied. By the arguments used in the proof of Theorem 4.1, we can see that these subgroups are indeed isomorphic to subgroups of $\operatorname{PGU}(3, q)$ fixing intersection sets of types $\mathcal{E}_{\text {III }}$ and $\mathcal{E}_{\text {IV }}$ of $\mathcal{H}_{1}$.

\section{Slices of the unitary spread of $\mathcal{Q}(6, q), q=3^{h}$}

Let $\mathbb{V}$ be the 8-dimensional vector space described in Sect. 2. If $q=3^{h}$, then the Quadratic form (1) on $\mathbb{V}$, defines a cone of the associated projective space $\mathbb{P}$ with vertex the point $\langle I\rangle_{\mathbb{F}_{q}}$ where $I$ is the identity matrix, and with base a parabolic quadric $\mathcal{Q}^{\prime}=\mathcal{Q}(6, q)$. We can choose as base of the cone the quadric contained in the hyperplane $\Pi$ with equation $\operatorname{Tr}(x)=0$; i.e. $\mathcal{Q}^{\prime}$ has equation

$$
-N(x)+\operatorname{Tr}(y z)+b c=0 .
$$

The set $\Omega$ exhibited in Sect. 2 then projects into an ovoid say $\Omega^{\prime}$ of $\mathcal{Q}^{\prime}$, indeed

$$
\left(\begin{array}{lll}
0 & 0 & 1 \\
0 & 0 & 0 \\
0 & 0 & 0
\end{array}\right) \text { and }\left(\begin{array}{ccc}
\alpha+\operatorname{Tr}(\alpha) & \alpha \beta^{q} & \alpha^{q+1} \\
\beta & 0 & \alpha^{q} \beta \\
1 & \beta^{q} & \alpha^{q}+\operatorname{Tr}(\alpha)
\end{array}\right)
$$

with $\alpha, \beta \in \mathbb{F}_{q^{2}}$ such that $\operatorname{Tr}(\alpha)+N(\beta)=0$.

In this section we classify the slices of the unitary spread $\mathcal{S}=\left\{T(X)^{\prime}: X \in \Omega\right\}$ of $\mathcal{Q}^{\prime}$ with respect to hyperplanes of $\Pi$ intersecting $\mathcal{Q}^{\prime}$ in elliptic quadrics, up to the action of $\overline{G_{\Pi}} \simeq \operatorname{PGU}(3, q) \rtimes C_{2}$. First, we prove the following

Theorem 5.1 Let $U$ be a hyperplane of $\Pi \simeq \mathrm{PG}(6, q)$, then the following possibilities can occur: 
1. $U$ is the polar hyperplane of a point of $\Omega^{\prime}$. There is a unique orbit of such hyperplanes; also, $U \cap \Omega^{\prime}$ is isomorphic to an intersection set $\mathcal{E}_{\mathrm{VI}}$.

2. $U$ is the polar hyperplane of a singular point not belonging to $\Omega^{\prime}$. There is a unique orbit of such hyperplanes; also, $U \cap \Omega^{\prime}$ is isomorphic to an intersection set $\mathcal{E}_{\mathrm{IV}}$.

3. $U$ is a non-singular hyperplane intersecting the $\mathcal{Q}(6, q)$ in a $\mathcal{Q}^{+}(5, q)$; such hyperplanes form a unique orbit; also, $U \cap \Omega^{\prime}$ is isomorphic to an intersection set $\mathcal{E}_{\mathrm{III}}$.

4. $U$ is a non-singular hyperplane intersecting the $\mathcal{Q}(6, q)$ in a $\mathcal{Q}^{-}(5, q)$; there are two orbits of such hyperplanes, say $\mathrm{O}_{1}$ and $\mathrm{O}_{2}$, the first of length $\frac{q^{3}\left(q^{2}-q+1\right)(q-1)}{6}$ and the second one of length $\frac{q^{3}(q+1)^{2}(q-1)}{3}$, such that for any $U \in O_{1}, U \cap \Omega^{\prime}$ is isomorphic to an intersection set $\mathcal{E}_{\mathrm{I}}$, while for any $U \in \mathrm{O}_{2}, U \cap \Omega^{\prime}$ is isomorphic to an intersection set $\mathcal{E}_{\mathrm{VII}}$.

Proof By Proposition 3.3, we know that the intersection $K \cap \Omega$ ( $K$ a hyperplane of $\mathbb{P}$ ) is isomorphic to the intersection of two Hermitian curves. Also, any hyperplane $U$ of $\Pi$ can be uniquely extended to a hyperplane $K$ of $\mathbb{P}$ passing through the vertex of $\mathcal{C}$ and $K \cap \Omega$ is isomorphic to $U \cap \Omega^{\prime}$.

If $U$ is a hyperplane polar of the point $P \in \Omega^{\prime}$, then we have that $U \cap \Omega^{\prime}$ is isomorphic to a subset $\mathcal{E}_{\mathrm{VI}}$ of $\mathcal{H}_{1}$. Consider, on the other hand,

$$
P=\left(\begin{array}{lll}
0 & 1 & 0 \\
0 & 0 & 1 \\
0 & 0 & 0
\end{array}\right)
$$

and denote by $\perp$ the polarity defined by the parabolic quadric $\mathcal{Q}(6, q)$, then $P \in$ $\mathcal{Q}(6, q) \backslash \Omega^{\prime}$ and $P^{\perp}$ has equation $\operatorname{Tr}(z)=0$. Arguing as in the proof of Theorem 4.1 we can prove that the intersection of this hyperplane with $\Omega^{\prime}$ is isomorphic to an intersection set $\mathcal{E}_{\mathrm{IV}}$ in $\mathcal{H}_{1}$. The subgroup of $\operatorname{PGU}(3, q)$ fixing such an intersection has order $q^{2}(q-1)$ (see [5]), hence the orbit of $U$ under the action of $G_{\Pi}$ has length $\frac{q^{3}\left(q^{3}+1\right)\left(q^{2}-1\right)}{q^{2}(q-1)}=q\left(q^{3}+1\right)(q+1)$.

So, we can state that any singular hyperplane which is the polar hyperplane of a point not in $\Omega^{\prime}$ intersects $\Omega^{\prime}$ in a set isomorphic to a set of type $\mathcal{E}_{\text {IV }}$ in $\mathcal{H}_{1}$. Slicing the unitary ovoid $\Omega^{\prime}$ with one of these singular hyperplanes we obtain a set of points which projects into a Kantor ovoid of $\mathcal{Q}(4, q)$ as already proven in [8]. Consider, now, the hyperplane $U$ of $\operatorname{PG}(6, q)$ defined by the following points

$$
\left\{\left(\begin{array}{ccc}
0 & y & c \\
z & 0 & y^{q} \\
b & z^{q} & 0
\end{array}\right): y, z \in \mathbb{F}_{q^{2}}, b, c \in \mathbb{F}_{q}\right\} .
$$

The intersection of $\mathcal{Q}$ with such a hyperplane is the hyperbolic quadric $\mathcal{Q}^{+}(5, q)$ of equation $\operatorname{Tr}(y z)+b c=0$. The points of $\Omega^{\prime} \cap U$ are:

$$
\left(\begin{array}{lll}
0 & 0 & 1 \\
0 & 0 & 0 \\
0 & 0 & 0
\end{array}\right) \text { and }\left(\begin{array}{ccc}
0 & \beta^{2 q+1} & \beta^{2(q+1)} \\
\beta & 0 & \beta^{2+q} \\
1 & \beta^{q} & 0
\end{array}\right)
$$


with $\beta \in \mathbb{F}_{q^{2}}$. Hence $\left|\Omega^{\prime} \cap U\right|=q^{2}+1$; since the unique orbit of hyperplanes, with respect to the action of $G_{\Pi}$, intersecting $\Omega^{\prime}$ in a set isomorphic to a $\mathcal{E}_{\text {IV }}$ consists of singular hyperplanes, the only possibility is that $U \cap \Omega^{\prime}$ is isomorphic to a set of type $\mathcal{E}_{\text {III }}$ and such hyperplanes form a unique orbit as well [8]. Let, now,

$$
U=\left\{\left(\begin{array}{ccc}
x & y & -b \\
z & 0 & y^{q} \\
b & z^{q} & x^{q}
\end{array}\right): x, y, z \in \mathbb{F}_{q^{2}}, x+x^{q}=0, b \in \mathbb{F}_{q}\right\}
$$

In this case, the intersection $U \cap \mathcal{Q}$ is the elliptic quadric $Q^{-}(5, q)$ with equation $x^{2}+\operatorname{Tr}(y z)-b^{2}=0$. The points of $\Omega^{\prime} \cap U$ are

$$
\left(\begin{array}{ccc}
\alpha+\operatorname{Tr}(\alpha) & \alpha \beta^{q} & -1 \\
\beta & 0 & \alpha^{q} \beta \\
1 & \beta^{q} & \alpha^{q}+\operatorname{Tr}(\alpha)
\end{array}\right)
$$

with $\alpha, \beta \in \mathbb{F}_{q^{2}}$ such that $\operatorname{Tr}(\alpha)+N(\beta)=0$ and $\alpha^{q+1}=-1$. So, $\left|U \cap \Omega^{\prime}\right|=(q+1)^{2}$ and by Proposition 3.6 the stabilizer of $U \cap \Omega^{\prime}$ is isomorphic to $\left(\left(\mathbb{Z}_{q+1} \times \mathbb{Z}_{q+1}\right) \rtimes\right.$ $\left.\mathrm{Sym}_{3}\right) \rtimes C_{2}$; indeed if this was not the case then we would have an orbit of such hyperplanes of length greater then the number of elliptic quadric in $Q(6, q)$. Hence, we have one orbit of length $\frac{q^{3}\left(q^{2}-q+1\right)(q-1)}{6}$, with respect to the action of $\overline{G_{\Pi}}$, of hyperplanes containing a $\mathcal{Q}^{-}(5, q)$ and intersecting $\Omega^{\prime}$ in a set isomorphic to a set $\mathcal{E}_{\mathrm{I}}$. There are, then, $\frac{q^{3}(q+1)^{2}(q-1)}{3}$ hyperplanes containing a $\mathcal{Q}^{-}(5, q)$ left and, by Lemma 3.5 , the only possibility is that they form one orbit and they intersect $\Omega^{\prime}$ in a set isomorphic to a $\mathcal{E}_{\mathrm{VII}}$.

Let $U$ be a hyperplane of $\Pi$ intersecting $\mathcal{Q}^{\prime}$ in a $\mathcal{Q}^{+}(5, q)$, then the set $\Omega^{\prime} \cap$ $\mathcal{Q}^{+}(5, q)$ is an ovoid of $\mathcal{Q}^{+}(5, q)$ and, by Theorem 5.1, it consists of $q-1$ pairwise disjoint conics and two special points. This set corresponds, via the Klein map, to a spread of the 3 -dimensional projective space $\mathrm{PG}(3, q)$ containing $q-1$ disjoint reguli and two special lines. In what follows, we explicitly describe such a spread. To this aim, consider the elliptic quadric $\mathcal{Q}^{+}(5, q)$ with equation $\operatorname{Tr}(y z)+b c=0$ and note that the set $\Omega^{\prime} \cap \mathcal{Q}^{+}(5, q)$ consists of the points:

$$
\left(\begin{array}{lll}
0 & 0 & 1 \\
0 & 0 & 0 \\
0 & 0 & 0
\end{array}\right) \text { and }\left(\begin{array}{ccc}
0 & \beta^{2 q+1} & \beta^{2(q+1)} \\
\beta & 0 & \beta^{2+q} \\
1 & \beta^{q} & 0
\end{array}\right)
$$

with $\beta \in \mathbb{F}_{q^{2}}$. Let $\xi$ be a fixed non-square element in $\mathbb{F}_{q}$. Then any element $x$ of $\mathbb{F}_{q^{2}}$ can be uniquely written as $x_{0}+x_{1} \sigma$, where $x_{0}, x_{1} \in \mathbb{F}_{q}$ and $\sigma^{2}=\xi$. Consider the following isomorphism $(y, z, b, c) \in \mathbb{F}_{q^{2}} \times \mathbb{F}_{q^{2}} \times \mathbb{F}_{q} \times \mathbb{F}_{q} \mapsto$ $\left(y_{0}, y_{1}, b, c,-z_{1} \xi,-z_{0}\right) \in \mathbb{F}_{q}^{6}$. Then, the equation $\operatorname{Tr}(y z)+b c=0$ can be written in the following way $-y_{0} z_{0}-y_{1} z_{1} \xi+b c=0$, and applying the above isomorphism it is isomorphic to the Klein quadric with equation $x_{1} x_{6}+x_{2} x_{5}+$ $x_{3} x_{4}=0$. In this setting, $\Omega^{\prime} \cap \mathcal{Q}^{+}(5, q)=\left\{P_{\infty}, P_{\beta}\right\}$ where $P_{\infty}=(0,0,0,1,0,0)$ and $P_{\beta}=\left(\beta^{q+1} \beta_{0},-\beta^{q+1} \beta_{1}, 1, \beta^{2(q+1)},-\beta_{1} \xi,-\beta_{0}\right), \beta \in \mathbb{F}_{q^{2}}$. Applying the inverse of the Klein map, we get: $P_{\infty} \mapsto \ell_{\infty}=\langle(0,1,0,0),(0,0,1,0)\rangle_{\mathbb{F}_{q}}$ and $P_{\beta} \mapsto$ 
$\ell_{\beta}=\left\langle\left(1, \beta_{1} \xi,-\beta_{0}, 0\right),\left(0, \beta_{0} \beta^{q+1},-\beta_{1} \beta^{q+1}, 1\right)\right\rangle_{\mathbb{F}_{q}}$. The set $\mathcal{L}=\left\{\ell_{\infty}, \ell_{\beta}\right\}, \beta \in \mathbb{F}_{q^{2}}$, is a spread of $\mathrm{PG}(3, q)$. Consider the hyperbolic quadrics $\mathcal{Q}_{d}:=\mathcal{Q}^{+}(3, q)$ with equation $x_{1}^{2} \xi d+x_{2}^{2}-x_{3}^{2} \xi-x_{4}^{2} d^{3}=0$, where $d$ is an element of $\mathbb{F}_{q}^{*}$. The line $\ell_{\beta}$ is contained in $\mathcal{Q}_{d}$ if and only if $\beta^{q+1}=d$, and hence the spread $\mathcal{L}$ contains $q-1$ disjoint reguli. The lines $\ell_{0}$ and $\ell_{\infty}$ are not contained in any of the $q-1$ quadrics but they are pairwise polar with respect to the polarity defined by $\mathcal{Q}_{d} \forall d \in \mathbb{F}_{q}^{*}$. This spread is spawned by a regular hyperbolic fibration of $\mathrm{PG}(3, q)$. Hyperbolic fibrations were introduced in [1] and in fact they consist of $q-1$ hyperbolic quadrics and two lines such that they form a partition of the point-set of $\mathrm{PG}(3, q)$; if the two lines are pairwise polar with respect to the polarity induced by any of the hyperbolic quadric, then the hyperbolic fibration is said to be regular. Choosing one regulus in each quadric, we get a line-spread of $\mathrm{PG}(3, q)$.

In [1, Theorem 2.2], the authors exhibit three families of regular hyperbolic fibrations. One of these is the following

$$
\mathcal{J}_{0}=\left\{V\left[t, 0,-\omega t^{p^{i}}, 1,0,-\omega\right]: t \in \mathbb{F}_{q}\right\} \cup\left\{l_{0}, l_{\infty}\right\}, \quad i \in\{0,1,2, \ldots, h\},
$$

where $\omega$ is a fixed non-square element in $\mathbb{F}_{q}$ and for any $t \in \mathbb{F}_{q}^{*}$

$$
V\left[t, 0,-\omega t^{p}, 1,0,-\omega\right]=t x_{1}^{2}-\omega t^{p^{i}} x_{2}^{2}+x_{3}^{2}-\omega x_{4}^{2} .
$$

Straightforward computations show that the hyperbolic fibration spawned by the spread $\mathcal{L}$ is isomorphic to the hyperbolic fibration $\mathcal{J}_{0}$ when $p=3$ and $i=1$.

The authors also find a linear automorphism group $\mathcal{G}$ in the stabilizer of $\mathcal{J}_{0}$; the group $\mathcal{G}$ has order $4\left(q^{2}-1\right)$ and is proven to be the semidirect product of a cyclic group of order $q^{2}-1$ and a Klein 4 -group. The subgroup $\mathcal{G}^{\prime}$ of $\mathcal{G}$ fixing $\mathcal{L}$ has order $2\left(q^{2}-1\right)$, since $\mathcal{G}^{\prime}$ does not contain the collineation of order two interchanging the two reguli of each hyperbolic quadric belonging to $\mathcal{J}_{0}$. Also, the authors state that MAGMA computations for $q=9$ show that the full linear stabilizer of $\mathcal{J}_{0}$ has order $8\left(q^{2}-1\right)$. As a consequence of Theorem 5.1 we have that the full linear stabilizer of $\mathcal{J}_{0}$ has always order at least $8\left(q^{2}-1\right)$. Indeed, by Theorem 5.1, the automorphism group of $\mathcal{L}$ is isomorphic to $\left(\operatorname{Aut}\left(\mathcal{E}_{\text {III }}\right) \cap \operatorname{PGU}(3, q)\right) \rtimes C_{2}$, where $\operatorname{Aut}\left(\mathcal{E}_{\text {III }}\right) \cap \operatorname{PGU}(3, q)$ is the semidirect product of a group of order two permuting the two special lines $l_{0}$ and $l_{\infty}$ and leaving the remaining invariant and a cyclic group of order $q^{2}-1$, acting regularly on the lines of the spread different from $l_{0}$ and $l_{\infty}$. The group $C_{2}$ fixes the lines $l_{0}$ and $l_{\infty}$ and fixes each regulus of the fibration. Moreover, $C_{2}$ fixes $\ell_{\beta}$ if and only if $\beta \in \mathbb{F}_{q}$ and this is possible if and only if $d$ is a square in $\mathbb{F}_{q}$; so, in $\frac{q-1}{2}$ reguli there are no fixed lines while in the remaining ones two fixed lines. Hence, the full linear stabilizer of $\mathcal{L}$ has size $4\left(q^{2}-1\right)$ and we can conclude that for any $q=3^{h}$, the full linear stabilizer of a regular fibration which belongs to the family $\mathcal{J}_{0}$ has order at least $8\left(q^{2}-1\right)$.

Now, let $U$ be a non-singular hyperplane intersecting $\mathcal{Q}$ in an elliptic quadric $\mathcal{Q}^{-}(5, q)$ : the intersection $U \cap \Omega^{\prime}$ is a partial ovoid of $\mathcal{Q}^{-}(5, q)$; on the other hand the intersection $U \cap \mathcal{S}$, where $\mathcal{S}=\left\{T(X)^{\prime}: X \in \Omega\right\}$, induces a spread say $\mathcal{S}^{\prime}$ of $\mathcal{Q}^{-}(5, q)$. Lemma 3.4 can be applied to see that the partial ovoid and the spread $\mathcal{S}^{\prime}$ have the same stabilizer. Also, in the previous theorem, we have pointed out that there are two 
types of non-singular hyperplanes intersecting $\mathcal{Q}(6, q)$ in a $\mathcal{Q}^{-}(5, q)$. Let $U \cap \Omega^{\prime}$ be isomorphic to a set $\mathcal{E}_{\mathrm{I}}$ in $\mathcal{H}_{1}$; the set $U \cap \Omega^{\prime}$ consists of $q+1$ pairwise disjoint conics. More precisely, taking into account the structure of the pencil of Hermitian curve of $\operatorname{PG}\left(2, q^{2}\right)$ with intersection set $\mathcal{E}_{\mathrm{I}}$, one can see that there are three different possible partitions of $U \cap \Omega^{\prime}$ into a set of $q+1$ disjoint conics, say $\left\{\mathcal{P}_{1}, \mathcal{P}_{2}, \mathcal{P}_{3}\right\}$. The subgroup of $\bar{H}$ fixing this set is isomorphic to $\left(\left(\mathbb{Z}_{q+1} \times \mathbb{Z}_{q+1}\right) \rtimes \mathrm{Sym}_{3}\right) \rtimes C_{2}$, where $\mathrm{Sym}_{3}$ is the symmetric group over three objects and $\mathbb{Z}_{q+1}$ is a cyclic group of order $q+1$; for any $i \in\{1,2,3\}$ one of the two copies of $\mathbb{Z}_{q+1}$ acts regularly on the conics of $\mathcal{P}_{i}$, the other one acts regularly on the points of the conics of $\mathcal{P}_{i}$. Moreover, the group $\operatorname{Sym}_{3}$ acts on the set $\left\{\mathcal{P}_{1}, \mathcal{P}_{2}, \mathcal{P}_{3}\right\}$. Now, looking at the action of this group on the spread $\mathcal{S}^{\prime}$ we have that it has one orbit of length $(q+1)^{2}$, which is in fact formed by the lines of $\mathcal{S}^{\prime}$ containing the points of $\mathcal{Q}^{-}(5, q) \cap \Omega^{\prime}$.

Finally, let $U$ be a hyperplane of $\Pi$ containing $\mathcal{Q}^{-}(5, q)$ such that $U \cap \Omega^{\prime}$ is isomorphic to an intersection set of type $\mathcal{E}_{\mathrm{VII}}$. In this case $\left|U \cap \Omega^{\prime}\right|=q^{2}-q+1$ and this set of points has the property that three of them are never contained in a conic. The automorphism group is isomorphic to $\operatorname{Aut}\left(\mathcal{E}_{\mathrm{VII}}\right) \rtimes C_{2}$ and acts transitively on the points of this partial ovoid and hence on the lines of the induced spread containing these points.

It is worth mentioning that since the generalized quadrangle $\mathcal{Q}^{-}(5, q)$ is isomorphic to the dual of the generalized quadrangle $\mathcal{H}\left(3, q^{2}\right)$ (for more details we refer to [13]), these two classes of spreads of $\mathcal{Q}^{-}(5, q)$ produce two non-isomorphic classes of ovoids of the Hermitian surface $\mathcal{H}\left(3, q^{2}\right)$ admitting $\left(\left(\mathbb{Z}_{q+1} \times \mathbb{Z}_{q+1}\right) \rtimes \mathrm{Sym}_{3}\right) \rtimes C_{2}$ and $\left(\mathbb{Z}_{3} \rtimes \mathbb{Z}_{q^{2}-q+1}\right) \rtimes C_{2}$.

\section{References}

1. Baker, R.D., Ebert, G.L., Wantz, K.L.: Regular hyperbolic fibrations. Adv. Geom. 1(2), 119-144 (2001)

2. Cooperstein, B.N.: Hyperplane sections of Kantor's unitary ovoid. Des. Codes Cryptogr. 23(2), 185195 (2001)

3. Dillon, J.F.: Elementary Hadamard difference sets. Ph.D. thesis, Univ. of Meryland, College Park (1974)

4. Dye, R.: Partitions and their stabilizers for line complexes and quadrics. Ann. Mat. Pura Appl. 114(4), 173-194 (1977)

5. Giuzzi, L.: Collineation groups of the intersection of two classical unitals. J. Comb. Des. 9(6), 445459 (2001)

6. Hirschfeld, J.W.P.: Finite Projective Spaces of Three Dimension. Oxford Science Publications. The Clarendon Press, Oxford University Press, New York (1985)

7. Hirschfeld, J.W.P., Thas, J.A.: General Galois Geometries. Oxford Science Publications. The Clarendon Press, Oxford University Press, New York (1991)

8. Kantor, W.M.: Ovoids and translation planes. Can. J. Math. 34(5), 1195-1207 (1982)

9. Kantor, W.M.: Spreads, translation planes and Kerdock sets I. SIAM J. Algebr. Discrete Methods 3(2), 151-165 (1982)

10. Kestenband, B.C.: Unital intersections in finite projective planes. Geom. Dedicata 11(1), 107-117 (1981)

11. Lunardon, G.: Normal spreads. Geom. Dedicata 75(3), 245-261 (1999)

12. Moisio, M.: Kloosterman sums, elliptic curves, and irreducible polynomials with prescribed trace and norm. Acta Arith. 132(4), 329-350 (2008)

13. Payne, S.E., Thas, J.A.: Finite Generalized Quadrangles. Pitman Advanced Publishing Program (1984) 
14. Taylor, D.E.: The Geometry of the Classical Groups. Sigma Series in Pure Mathematics, vol. 9. Heldermann, Berlin (1992)

15. Thas, J.A.: Polar spaces, generalized hexagons and perfect codes. J. Comb. Theory (A) 29(1), 87-93 (1980)

16. Tits, J.: Sur la trialité et certains groupes qui s'en déduisent. Inst. Hautes Etudes Sci. Publ. Math., 13-60 (1959)

17. Van Maldeghem, H.: Generalized Polygons. Birkhäuser, Basel (1998) 